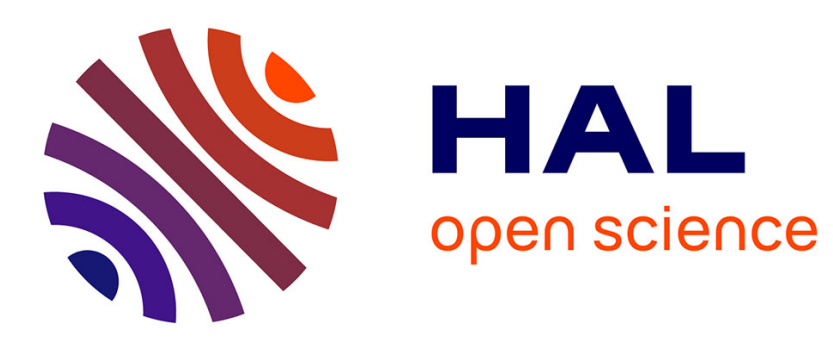

\title{
From preferences over arguments to preferences over attacks in abstract argumentation: A comparative study
}

Claudette Cayrol, Marie-Christine Lagasquie-Schiex

\section{To cite this version:}

Claudette Cayrol, Marie-Christine Lagasquie-Schiex. From preferences over arguments to preferences over attacks in abstract argumentation: A comparative study. 25th International Conference on Tools with Artificial Intelligence - ICTAI 2013, Nov 2013, Washington, DC, United States. pp. 588-595. hal-01148809

\section{HAL Id: hal-01148809 \\ https://hal.science/hal-01148809}

Submitted on 5 May 2015

HAL is a multi-disciplinary open access archive for the deposit and dissemination of scientific research documents, whether they are published or not. The documents may come from teaching and research institutions in France or abroad, or from public or private research centers.
L'archive ouverte pluridisciplinaire HAL, est destinée au dépôt et à la diffusion de documents scientifiques de niveau recherche, publiés ou non, émanant des établissements d'enseignement et de recherche français ou étrangers, des laboratoires publics ou privés. 


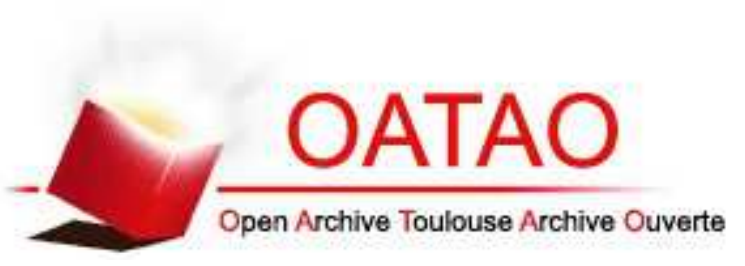

\section{Open Archive TOULOUSE Archive Ouverte (OATAO)}

OATAO is an open access repository that collects the work of Toulouse researchers and makes it freely available over the web where possible.

This is an author-deposited version published in : http://oatao.univ-toulouse.fr/ Eprints ID : 12762

To link to this article : DOI: $\underline{10.1109 / I C T A I .2013 .93}$

URL : http://dx.doi.org/10.1109/ICTAI.2013.93

To cite this version : Cayrol, Claudette and Lagasquie-Schiex, MarieChristine From preferences over arguments to preferences over attacks in abstract argumentation: A comparative study. (2013) In: 25th International Conference on Tools with Artificial Intelligence ICTAI 2013, 4 November 2013 - 6 November 2013 (Washington, DC, United States).

Any correspondance concerning this service should be sent to the repository administrator: staff-oatao@ listes-diff.inp-toulouse.fr 


\title{
From preferences over arguments to preferences over attacks in abstract argumentation: A comparative study
}

\author{
Claudette Cayrol, Marie-Christine Lagasquie-Schiex \\ IRIT-UPS, University of Toulouse, France \\ Email: \{ccayrol, lagasq\}@irit.fr
}

\begin{abstract}
Dung's argumentation framework has been extended to consider preferences over arguments or over attacks, in a qualitative or in a quantitative way. In this paper, we investigate the relationships between preferences over arguments and preferences over attacks. We give conditions on the definition of preferences over attacks from preferences over arguments. Following these principles, we propose different instantiations of an $A F_{V S}$ (argumentation framework with attacks of various strength), when preferences over arguments are available. Our proposal is compared to existing work, particularly regarding the conditions in which the defence holds.
\end{abstract}

Keywords-Reasoning Agents, Knowledge Representation, Argumentation.

\section{INTRODUCTION}

Argumentation has become an influential approach in Artificial Intelligence to model cognitive tasks such as inconsistency handling and defeasible reasoning (e.g. [1], [2], [3]), decision making (e.g. [4]), or negotiation between agents (e.g. [5]). It is based on the evaluation of interacting arguments, which may support opinions, claims or decisions. Usually, the interaction takes the form of conflicts between arguments, and the fundamental issue is the selection of acceptable sets of arguments, based on the way they interact and the key concept of defence. Most of the argumentationbased proposals are instantiations of the abstract system proposed by Dung [6], which is reduced to a set of arguments (completely abstract entities) and a binary relation, called attack, which captures the conflicts between arguments. The increasing interest for the argumentation formalism has led to numerous extensions of the basic abstract system which are more appropriate to the applications.

A first extension of Dung's system has included a preference relation between arguments, which models their relative strength. For instance, an argument built from certain knowledge is stronger than an argument relying upon default knowledge (see e.g. [7], [8], [9], [10]).

Other extensions have been proposed to make a distinction between various types of conflict: [11], [12] in a logical setting, [13] in case of symmetric attacks, [14] in a multiagent setting, [15], [16] with weighted attacks. Behind these proposals, there is a common idea that attacks may have different strength and can be compared according to their relative strength. However, there is so far no consensus about how it should be used to define extensional semantics, according to which acceptable sets of arguments are selected. Some works towards that direction have been proposed in [17], [18], [19], where an abstract argumentation system with varied-strength attacks has been defined and a new kind of defence, the vs-defence, has been introduced.

Our purpose is to take advantage of that abstract system in order to investigate the relationships between preferences over arguments and preferences over attacks. We consider quantitative as well as qualitative approaches for expressing these preferences. Some results for the quantitative setting have been presented in [20]. We pursue that work by providing a comparative study of different proposals. Sect. II gives the background about the modelling of strength (of arguments and of attacks) in abstract argumentation. Sect. III presents our proposals for moving from preferences over arguments to preferences over attacks. These proposals are compared with existing ones in Sect. IV and Sect. V is devoted to concluding remarks.

\section{BACKGROUND}

\section{A. Dung's argumentation framework}

In Dung's abstract framework [6], arguments are supposed to be given and conflicts between arguments are represented by a binary attack relation.

Def. 1: An argumentation framework (AF) is a pair $\langle\mathbf{A}, \mathbf{R}\rangle$ of a finite set $\mathbf{A}$ of arguments ${ }^{1}$ and a binary (attack) relation $\mathbf{R}$ over $\mathbf{A}$. $A \mathbf{R} B$ means that $A$ attacks $B$.

An AF may be represented by a directed graph $\mathcal{G}$ whose nodes represent arguments and edges represent the attack relation. Acceptable sets of arguments can be defined from the concepts of conflict-freeness and defence.

Def. 2: A set $\mathcal{S} \subseteq \mathbf{A}$ is conflict-free iff $^{2}$ there are no $A, B \in \mathcal{S}$ such that $A \mathbf{R} B$. Let $A, B, C \in \mathbf{A}, C$ defends $A$ against $B$ iff $B \mathbf{R} A$ and $C \mathbf{R} B$. A set $\mathcal{S}$ defends $A$ iff for each $B$ such that $B \mathbf{R} A$, there exists $C \in \mathcal{S}$ such that $C$ defends $A$ against $B . \mathcal{S}$ is admissible iff $\mathcal{S}$ is conflict-free and defends all its elements.

\footnotetext{
${ }^{1}$ We assume that $\mathbf{A}$ represents the set of arguments proposed by rational agents at a given time; so it makes sense to assume that $\mathbf{A}$ is finite.

2 iff: if and only if
} 


\section{B. Preferences between arguments: PAF or WAF}

Strengths of arguments have been introduced in two ways:

- a qualitative way in Preference Argumentation Frameworks (PAF) [3]: an AF is associated with a partial preordering $\succeq$ over $\mathbf{A}$; so a $\mathrm{PAF}$ is a triplet $\langle\mathbf{A}, \mathbf{R}, \succeq\rangle$.

- a quantitative way in Weighted Argumentation Frameworks (WAF) [16]: an AF is associated with a function $\omega: \mathbf{A} \rightarrow \mathbb{R}$; so a WAF is also a triplet $\langle\mathbf{A}, \mathbf{R}, \omega\rangle$.

Notations: Let $\succeq$ be a binary relation on $\mathbf{A}$.

- The corresponding strict relation is denoted by $\succ$ and defined by $A \succ B$ iff ( $A \succeq B$ and $\operatorname{not}(B \succeq A)$ ). Note that $\succ$ is irreflexive and asymmetric.

- $A \sim B$ denotes $(A \succeq B$ and $B \succeq A$ ); it means that $A$ and $B$ are equivalent.

- $A \not B$ denotes $(\operatorname{not}(A \succeq B)$ and $\operatorname{not}(B \succeq A))$; it means that $A$ and $B$ are incomparable.

A WAF enables to construct a particular PAF as follows:

Def. 3: Let WAF $=\langle\mathbf{A}, \mathbf{R}, \omega\rangle$. The PAF corresponding to WAF is the triplet $\langle\mathbf{A}, \mathbf{R}, \succeq\rangle$ where $\forall A, B \in \mathbf{A}, A \succeq B$ iff $\omega(A) \geq \omega(B)$.

In this case, the resulting preordering $\succeq$ is total (no incomparable arguments in the PAF).

\section{Preferences between attacks}

Strengths of attacks have also been introduced in a qualitative way and in a quantitative way. The first modelling is given in [18], [19] (a variant has been introduced in [17]):

Def. 4: An argumentation framework with attacks of various strength $\left(\mathrm{AF}_{\mathrm{Vs}}\right)$ is a triplet $\langle\mathbf{A}, \mathbf{A T T}, \overleftrightarrow{\succeq}\rangle$ where $\mathbf{A}$ is a finite set of arguments, ATT is a finite set of attack relations $\{\stackrel{1}{\rightarrow}, \ldots, \stackrel{n}{\rightarrow}\}$ over $\mathbf{A}$ and $\overleftrightarrow{\succeq}$ is a binary relation over ATT.

Each $\stackrel{i}{\rightarrow} \subseteq \mathbf{A} \times \mathbf{A}$ represents a conflict relation, and $\vec{\succeq}$ enables to express a relative strength between these relations. The relation $\succeq$ is only assumed reflexive (it may be partial, and transitive or not). The corresponding strict relation, equivalence relation and incomparability relation will be respectively denoted by $\vec{\succ}, \vec{\sim}$, and $\vec{z}$.

In [18], [19], the strength of attacks has been used for refining the notion of defence. When $B \mathbf{R} A$ and $C \mathbf{R} B$, it is natural to account for the relative strength of the attacks in order to define the defence of $A$ by $C$. If the attack on $B$ is too weak to inhibit the attack on $A$, it will not be relevant for reinstating $A$. Let $\mathrm{AF}_{\mathrm{Vs}}=\langle\mathbf{A}, \mathbf{A T T}, \succeq\rangle$, the following definition captures the idea of relevant defender ${ }^{3}$ :

Def. 5: Let $A, B, C \in \mathbf{A}$ such that $C \stackrel{j}{\rightarrow} B$ and $B \stackrel{i}{\rightarrow} A$. $C$ vs-defends $A$ against $B$ (or $C$ is a vs-defender ${ }^{4}$ of $A$ against $B$ ) iff $\stackrel{i}{\rightarrow} \underset{\nsucc}{\rightarrow} \rightarrow$ (i.e. the attack from $B$ to $A$ is not strictly stronger than the one from $C$ to $B$ ).

\footnotetext{
${ }^{3}$ Note that if $\vec{\succ}$ is empty, the classical notion of defence is recovered.

${ }^{4}$ vs means "various strength".
}

The second modelling is given in [20]. It is a quantitative approach inspired by [16].

Def. 6: An argumentation framework with variedstrength defeat (AFV) is a triplet $\langle\mathbf{A}, \mathbf{R}, \mathrm{Vdef}\rangle^{5}$ where $\mathbf{A}$ is a finite set of arguments, $\mathbf{R}$ is an attack relation and Vdef is a function from $\mathbf{R}$ to the interval $[0,1]$. $\operatorname{Vdef}(A, B)$ represents the degree of certainty of the statement " $A$ attacks $B$ ".

Note that, according to the previous definition, [20] considers that the case when $\operatorname{Vdef}(A, B)=0$ is equivalent to $\operatorname{not}(A$ attacks $B)$. In other words, attacks that have a Vdef value equal to 0 are removed from $\mathbf{R}$.

A new notion of defence is defined in an AFV:

Def. 7: Let $A, B, C \in \mathbf{A}$ such that $C \mathbf{R} B$ and $B \mathbf{R} A$ (so with $\operatorname{Vdef}(C, B) \neq 0$ and $\operatorname{Vdef}(B, A) \neq 0$ ). $C$ vdefdefends $A$ against $B$ (or $C$ is a vdef-defender of $A$ against $B)$ iff $\operatorname{Vdef}(C, B) \geq \operatorname{Vdef}(B, A)$ (i.e. the attack from $C$ to $B$ is at least as strong as the attack from $B$ to $A$ ).

Note that the intuition for extending the notion of defence is the same in both frameworks $A F_{V S}$ and AFV. The only difference lies in the nature of the preference relation over attacks: an $A_{\text {vs }}$ uses a preordering (allowing for incomparable attacks), whereas in an AFV the preference relation is based on a function with values on a linearly ordered scale (thus providing a total ordering over attacks).

\section{From preferences between arguments to weighted attacks}

In [20], different instantiations of an $\mathrm{AFV}^{6}$ by a PAF or a WAF have been proposed, according to the following intuition: the larger the preference of $A$ over $B$, the stronger the attack from $A$ to $B$.

Def. 8: Let PAF $=\langle\mathbf{A}, \mathbf{R}, \succeq\rangle$. Two different AFV $=$ $\langle\mathbf{A}, \mathbf{R}, \mathrm{Vdef}\rangle$ can be built according to the following definitions for Vdef: let $(A, B) \in \mathbf{R}$,

- $\operatorname{Vdef}_{1}^{\succ}(A, B)=0$ if $B \succ A, 1$ otherwise

- $\operatorname{Vdef}_{2}^{\succ}(A, B)=0$ if $B \succ A, 1$ if $A \succ B, 1 / 2$ otherwise

Def. 9: Let WAF $=\langle\mathbf{A}, \mathbf{R}, \omega\rangle$. Three different $A F V=$ $\langle\mathbf{A}, \mathbf{R}, \mathrm{Vdef}\rangle$ can be built according to the following definitions for Vdef: let $(A, B) \in \mathbf{R}$,

- Vdef $\mathrm{f}_{\text {bool }}^{\omega}(A, B)=0$ if $\omega(B)>\omega(A), 1$ otherwise

- $\operatorname{Vdef}_{1}^{\omega}(A, B)=\max (\omega(A)-\omega(B), 0)$

- $\operatorname{Vdef} f_{2}^{\omega}(A, B)=1-\max (\omega(B)-\omega(A), 0)$

Note that $\operatorname{Vde} f_{\text {bool }}^{\omega}$ exactly corresponds to $\operatorname{Vde} f_{1}^{\succ}$, when $\succ$ is built from $\omega$ as in Def.3.

Similarly, Vdef $\mathrm{f}_{2}^{\succ}$ could be built from a WAF by:

$\operatorname{Vdef}_{2}^{\succ}(A, B)=0$ if $\omega(B)>\omega(A), 1$ if $\omega(A)>\omega(B)$, $1 / 2$ otherwise.

These instantiations of an AFV have been extensively studied in [20] and interesting properties have been provided,

\footnotetext{
${ }^{5}$ def stands for defeat.

${ }^{6}$ In [20], these instantiations have been built using an intermediary argumentation framework, called a VPAF. Nevertheless, due to lack of place, here we only give the last step of this process.
} 
particularly for $V$ def $f_{2}^{\omega}$. However, most of the definitions for Vdef lead to suppress the attacks $(A, B)$ for which $B$ is strictly preferred to $A$. With Vde $\mathrm{f}_{2}^{\omega}$, an attack from $A$ to $B$ is removed when $(\omega(B)-\omega(A))=1$.

This point is problematic to us. Moreover, our idea is to investigate the instantiation process in the qualitative setting.

\section{FROM PREFERENCES OVER ARGUMENTS TO PREFERENCES OVER ATTACKS}

Our purpose is to investigate different ways by which preferences over attacks are computed from preferences over arguments. In the qualitative setting (Sect. III-A), we start by laying down some principles which should govern the instantiation of an $A_{\text {Vs }}$ by a PAF and we propose examples of such an instantiation. The partial preordering and the attack relation available in a PAF serve to compute a particular $A F_{V s}$ in which the relative strength of attacks is represented by a preordering on the attacks. A particular case is to start from a WAF and to construct the associated PAF according to Def.3. Then, we consider a quantitative setting (Sect. III-B) and follow the same methodology. We propose some principles and take advantage of weights over arguments in order to define a value-based preference on the attacks. An important result is that we can provide a construction of a valued $A F_{V s}$ in which the preference over attacks refines the preference obtained in the qualitative setting (see Sect.III-C).

\section{A. $\mathrm{AF}_{\mathrm{Vs}}$ induced by a PAF}

We introduce some conditions on the relationship between $\mathrm{PAF}=\langle\mathbf{A}, \mathbf{R}, \succeq\rangle$ and $\mathrm{AF}_{\mathrm{VS}}=\langle\mathbf{A}, \mathbf{A T T}, \underset{\succeq}{\overrightarrow{\mathrm{T}}}\rangle$ when ATT and $\succeq$ are built from $\mathbf{R}$ and $\succeq$. These conditions are expressed under the form of principles:

- P1 The initial set of conflicts between arguments must not be modified (no attack disappears, no attack appears).

- P2 An attack from $A$ to $B$ with $A$ strictly preferred to $B$ must be strictly stronger than an attack between two equivalent or incomparable arguments; in the same way, an attack from $A$ to $B$ with $B$ strictly preferred to $A$ must be strictly weaker than an attack between two equivalent or incomparable arguments; this principle induces a partition into at least 3 classes of attacks.

- P3 An attack between two equivalent arguments must be strictly stronger than an attack between two incomparable arguments; this principle associated with $\mathbf{P 2}$ induces a partition into at least 4 classes of attacks.

So, starting from PAF $=\langle\mathbf{A}, \mathbf{R}, \succeq\rangle$, the following definitions give the way for building an $A F_{V S}$ respecting P1 to P3:

Def. 10: The partition of $\mathbf{R}$ induced by PAF, denoted by $\operatorname{Part}(\mathbf{R})$, is defined as the set $\{\stackrel{1}{\rightarrow}, \stackrel{2}{\rightarrow}, \stackrel{3}{\rightarrow}, \stackrel{4}{\rightarrow}\}$ with:

- $\stackrel{1}{\rightarrow}=\{(A, B) \in \mathbf{R}$ such that $A \succ B\}$
- $\stackrel{2}{\rightarrow}=\{(A, B) \in \mathbf{R}$ such that $A \sim B\}$

- $\stackrel{3}{\rightarrow}=\{(A, B) \in \mathbf{R}$ such that $A \not \approx B\}$

- $\stackrel{4}{\rightarrow}=\{(A, B) \in \mathbf{R}$ such that $B \succ A\}$

Def. 11: The AF $\mathrm{Vs}_{\mathrm{VS}}$ induced by PAF is the triplet $\langle\mathbf{A}, \mathbf{A T T}, \succeq\rangle$ where $\mathbf{A T T}=\operatorname{Part}(\mathbf{R})$ and $\succeq$ is the reflexive transitive closure of the binary relation over $\operatorname{Part}(\mathbf{R})$ defined by: $\stackrel{1}{\rightarrow} \underset{\succ}{\rightarrow} \stackrel{2}{\rightarrow} \stackrel{3}{\rightarrow} \underset{\longrightarrow}{\rightarrow} \stackrel{4}{\rightarrow}$.

Note that the relation $\succeq$ is a total ordering over ATT. From the above definitions, it follows easily that $\vec{\succeq}$ enables to define preorderings on $\mathbf{R}$ that satisfy $\mathbf{P 1}, \mathbf{P 2}, \mathbf{P 3}$. It may be a total preordering such that two attacks of the same class are equivalent, or a partial preordering such that two attacks of the same class are incomparable.

Two other principles may be introduced in the qualitative setting for taking into account the following cases:

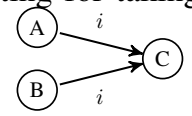
with $A \succ B$

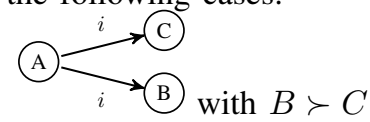

In these cases, it is natural to consider that:

$$
A \stackrel{i}{\rightarrow} C \stackrel{\vec{\succ}}{ } B \stackrel{i}{\rightarrow} C \quad \quad A \stackrel{i}{\rightarrow} C \stackrel{\vec{\succ}}{\rightarrow} A \stackrel{i}{\rightarrow} B
$$

Formally, we introduce ${ }^{7}$ :

- P4 Consider an attack from $A$ to $C$ and an attack from $B$ to $C$ of the same class. If $A$ is strictly stronger than $B$, the attack $(A, C)$ must be strictly stronger than the attack $(B, C)$.

- P5 Consider an attack from $A$ to $C$ and an attack from $A$ to $B$ of the same class. If $B$ is strictly stronger than $C$, the attack $(A, C)$ must be strictly stronger than the attack $(A, B)$.

So, starting from PAF $=\langle\mathbf{A}, \mathbf{R}, \succeq\rangle$, we define a new relation over $\mathbf{R}$, which is only a partial preordering:

Def. 12: Given Part $(\mathbf{R})$, the partial ordering $\succeq$ over $\mathbf{R}$ induced by $\mathbf{P 1}$ to $\mathbf{P 5}$ is the reflexive transitive closure of the binary relation over $\mathbf{R}$ defined by:

- Let $(A, B),(C, D) \in \mathbf{R}$ such that $(A, B) \in \stackrel{i}{\rightarrow}$ and $(C, D) \in \stackrel{j}{\rightarrow}$. Then $(A, B) \vec{\succ}(C, D)$ iff $i<j$.

- Let $(A, B),(C, D) \in \stackrel{2}{\rightarrow}$, then $(A, B) \stackrel{\sim}{\sim}(C, D)$.

- Let $(A, C),(B, C) \in \stackrel{1}{\rightarrow}$ (resp. $\stackrel{3}{\rightarrow}, \stackrel{4}{\rightarrow})$. If $A \succ B$ then $(A, C) \vec{\succ}(B, C)$, and if $A \sim B$ then $(A, C) \vec{\sim}(B, C)$.

- Let $(A, C),(A, B) \in \stackrel{1}{\rightarrow}$ (resp. $\stackrel{3}{\rightarrow}, \stackrel{4}{\rightarrow})$. If $B \succ C$ then $(A, C) \vec{\succ}(A, B)$, and if $B \sim C$ then $(A, C) \vec{\sim}(A, B)$.

Now, using this relation $\succeq$ on $\mathbf{R}^{8}$, we can define an $\mathrm{AF}_{\mathrm{Vs}}$ respecting $\mathbf{P 1}$ to $\mathbf{P 5}$.

Def. 13: The AF $\mathrm{Vs}_{\mathrm{VS}}$ induced by $\mathrm{PAF}$ is the triplet $\langle\mathbf{A}, \mathbf{A T T}, \vec{\succeq}\rangle$ where ATT is the set of singletons $\{(A, B)\}$ s.t. $(A, B) \in \mathbf{R}$ and $\succeq$ as defined in Def.12.

\footnotetext{
${ }^{7}$ Note that these principles do not apply to the class $\stackrel{2}{\rightarrow}$, since all tracks in this class are between arguments which are equivalent.

$8 \vec{\succeq}$ is partial since, considering $(A, B)$ and $(C, D) \in \stackrel{i}{\rightarrow}, \forall i=1,3,4$, with $A \neq C$ and $B \neq D$, there is no reason for deciding either that $(A, B) \vec{\succ}(C, D)$ or that $(A, B) \vec{\sim}(C, D)$.
} 
Note that a particular case of the construction provided by Def.11 (resp. Def.13) occurs when the PAF is obtained from a WAF according to Def.3.

In the following, we give some properties of $A F_{V S}$ induced by a PAF. First, it is worth noticing that an $A F_{V S}$ is a strict extension of a classical Dung's framework.

Let $\mathrm{AF}_{\mathrm{Vs}}=\langle\mathbf{A},\{\stackrel{1}{\rightarrow}, \ldots, \stackrel{n}{\rightarrow}\}, \overleftrightarrow{\succeq}\rangle, \mathrm{AF}=\langle\mathbf{A}, \cup \stackrel{i}{\rightarrow}\rangle$ denotes the classical Dung's argumentation framework associated with $A F_{V s}$. It has been shown in [18], [19] that if a set of arguments $\mathcal{S}$ is vs-admissible ${ }^{9}$ in $\mathrm{AF}_{\mathrm{VS}}$, it is also admissible in AF. However, there does not exist a formal compilation of an $A F_{V S}$ (even obtained from a PAF) into a classical Dung argumentation framework over the same set of arguments, which would be equivalent; i.e. such that the vs-admissible sets of $A F_{V s}$ would exactly coincide with the admissible sets of AF (see the following example).

Ex. 1: Consider the $A F_{\mathrm{Vs}}$ represented by:

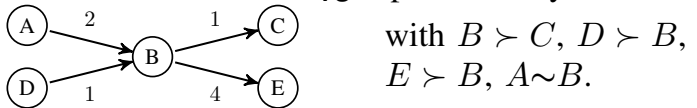

Assuming that $\mathrm{AF}_{\mathrm{Vs}}$ can be compiled into an equivalent classical $\langle\mathbf{A}, \mathbf{R}\rangle$, the following constraints hold:

- $\{A, C, D, E\}$ is vs-admissible, so $\{A, C, D, E\}$ must be admissible in $\langle\mathbf{A}, \mathbf{R}\rangle$, and so $\{A, C, D, E\}$ must be conflict-free in $\langle\mathbf{A}, \mathbf{R}\rangle$.

- $\{E\}$ is not vs-admissible, so $\{E\}$ should not be admissible in $\langle\mathbf{A}, \mathbf{R}\rangle$. So, $E$ must be attacked and since $\{A, C, D, E\}$ is conflict-free, $E$ is attacked by $B$ in $\langle\mathbf{A}, \mathbf{R}\rangle$, and $B$ is the only attacker of $E$ in $\langle\mathbf{A}, \mathbf{R}\rangle$.

- And similarly, $B$ is the only attacker of $C$ in $\langle\mathbf{A}, \mathbf{R}\rangle$.

- $\{A, E\}$ is vs-admissible, so $\{A, E\}$ must be admissible in $\langle\mathbf{A}, \mathbf{R}\rangle$. So, $A$ must attack $B$ in $\langle\mathbf{A}, \mathbf{R}\rangle$.

- $\{A, C\}$ is not a vs-admissible set, so $\{A, C\}$ should not be admissible in $\langle\mathbf{A}, \mathbf{R}\rangle$.

As $A$ attacks $B$, which is the only attacker of $C$, and due to the fact that $\{A, C, D, E\}$ is conflict-free, it must be the case that $\{A, C\}$ does not defend $A$. The only possibility is that $A$ is attacked by $B$ (since $\{A, C, D, E\}$ is conflict-free). But then, the set $\{A, C\}$ would still be admissible (since $A$ attacks $B$ ). So, in conclusion, it is not possible to turn the above $A F_{\text {vs }}$ into an equivalent classical system.

Nevertheless, there exist at least two cases in which the vs-defence in $A F_{V s}$ and the defence in $A F$ coincide:

- If all arguments are equivalent (resp. incomparable) then $\mathrm{AF}_{\mathrm{Vs}}=\mathrm{AF}$.

- If any attack from $A$ to $B$ is such that $A \succ B$ and if the preordering between arguments is a total one then $\mathrm{AF}_{\mathrm{Vs}}=\mathrm{AF}$.

Depending on the properties of the relations $\mathbf{R}$ and $\succeq$ over arguments, particular cases of Def.10 can be identified:

\footnotetext{
${ }^{9} \mathcal{S}$ is vs-admissible iff it is conflict-free and contains a vs-defender for each of its elements, see [18], [19].
}

- If $\succeq$ is a total preordering then there are only 3 classes of attacks in $\operatorname{Part}(\mathbf{R}): \stackrel{1}{\rightarrow}, \stackrel{2}{\rightarrow}$ and $\stackrel{4}{\rightarrow}$ (for instance, if the PAF is obtained from a WAF).

- If there is no $(A, B) \in \mathbf{R}$ such that $B \succ A$ then there are only 3 classes of attacks in $\operatorname{Part}(\mathbf{R}): \stackrel{1}{\rightarrow}$ to $\stackrel{3}{\rightarrow}$.

- If there is no $(A, B) \in \mathbf{R}$ such that $B \succ A$ and if $\succeq$ is a total preordering then there are only 2 classes of attacks in $\operatorname{Part}(\mathbf{R}): \stackrel{1}{\rightarrow}$ and $\stackrel{2}{\rightarrow}$.

Finally, we establish links between our construction and other approaches based on a framework close to $A F_{V s}$. The first one [13] starts from a PAF where the attack relation $\mathbf{R}$ is supposed symmetric. So, 3 classes of attack are obtained:

- $\mathbf{A T T}_{1}=\{(A, B)$ in conflict, with $A \succ B\}$ ( $A$ is a "proper defeater" of $B$ ),

- $\mathbf{A T T}_{2}=\{(A, B)$ in conflict, with $A \sim B\}$ ( $A$ is a "blocking defeater" of $B$ and vice-versa),

- $\mathbf{A T T}_{3}=\{(A, B)$ in conflict, with $A \not \approx B\}$ ( $A$ is a "blocking defeater" of $B$ and vice-versa).

The correspondence with our AF follows easily: let $(A, B)$ such that $A$ attacks $B$,

- $(A, B) \in \mathbf{A} \mathbf{T T}_{1}$ iff $(A, B) \in \stackrel{1}{\rightarrow}$ and $(B, A) \in \stackrel{4}{\rightarrow}$.

- $(A, B) \in \mathbf{A} \mathbf{T T}_{2}$ iff $(A, B)$ and $(B, A) \in \stackrel{2}{\rightarrow}$.

- $(A, B) \in \mathbf{A} \mathbf{T T}_{3}$ iff $(A, B)$ and $(B, A) \in \stackrel{3}{\rightarrow}$.

Note that a constraint analogous to $\mathbf{P 4}$ was added in [13] in order to compare two vs-defenders of a same argument.

Another approach given in [17] aims at defining four notions of defence in an $\mathrm{AF}_{\mathrm{Vs}}$ : let $C \stackrel{j}{\rightarrow} B \stackrel{i}{\rightarrow} A$,

- $C$ is a strong defender of $A$ against $B$ iff $\stackrel{j}{\rightarrow} \vec{\succ} \rightarrow$.

- $C$ is a weak defender of $A$ against $B$ iff $\stackrel{i}{\rightarrow} \vec{\succ} \rightarrow$.

- $C$ is a normal defender of $A$ against $B$ iff $\stackrel{i}{\rightarrow} \vec{\sim} \rightarrow$.

- $C$ is an unqualified defender of $A$ against $B$ iff $\stackrel{i}{\rightarrow} \not{z} \stackrel{j}{\rightarrow}$. It is easy to see that our notion of vs-defence corresponds to a defence which is either strong or normal or unqualified. Moreover, if we consider an $\mathrm{AF}_{\mathrm{Vs}}$ built from a PAF obtained with a WAF, the vs-defence corresponds to strong or normal defence.

\section{B. Valued $\mathrm{AF}_{\mathrm{Vs}}$ induced by a WAF}

In this section, we turn to the qualitative setting. Starting from a WAF, our purpose is to take advantage of the weights over arguments to define an $A F_{\text {vs }}$ with a valuebased preference over attacks. For simplicity, we assume that $\omega: \mathbf{A} \rightarrow[0,1]$, but any linearly ordered scale with top and bottom could be used as well.

Given $\omega$, we define the strength of an attack from $A$ to $B$ from $\omega$ and a function $f:[0,1] \times[0,1] \rightarrow \mathbb{R}$. Formally, let $A, B \in \mathbf{A}$, such that $A$ attacks $B$. We assume that the strength of the attack from $A$ to $B$ is quantified by $f(\omega(A), \omega(B))$. As for the construction of an $\mathrm{AF}_{\mathrm{Vs}}$ induced by a PAF, we want to impose conditions on the relationship between the weights on arguments and the strength on 
attacks. We keep Principles P1 and P2. Principle P3 becomes out of interest since there are no more incomparable arguments. Then we strengthen Principles P4 and P5 and reformulate them in terms of weights: let $(A, B) \in \mathbf{R}$,

- P4' If the weight of $A$ is greater than the weight of $B$, then the higher the difference of the weights, the stronger the attack from $A$ to $B$.

- P5 $^{\prime}$ If the weight of $A$ is lower than the weight of $B$, then the higher the difference of the weights, the weaker the attack from $A$ to $B$.

In order to build a valued AF ${ }_{\mathrm{Vs}}$ satisfying P1, P2, P4', $\mathbf{P 5}^{\prime}$, we assume some conditions on the function $f$.

Def. 14: A weighting translation function is a function $f:[0,1] \times[0,1] \rightarrow \mathbb{R}$ such that: $\forall x, y, z, t \in[0,1]$,

- if $x>y$ and $t>z$ then $f(x, y)>f(y, y)>f(z, t)$.

- $f(x, x)=f(y, y)$.

- if $x-y>z-t>0$, then $f(x, y)>f(z, t)$.

- if $x-y<z-t<0$, then $f(x, y)<f(z, t)$.

The first and second items of the previous definition allow the respect of $\mathbf{P 2}$. The other items correspond to $\mathbf{P 4}{ }^{\prime}$ and $\mathbf{P 5}^{\prime}$.

An example of a such weighting translation function is:

Def. 15: $\forall x, y \in[0,1], f_{\alpha \beta}(x, y)=\alpha(x-y)+\beta$, with $\alpha>0$ and $\beta>0$.

The following definition gives the way for building an AF Vs $_{\text {respecting }} \mathbf{P 1}, \mathbf{P 2}, \mathbf{P 4}{ }^{\prime}, \mathbf{P 5}^{\prime}$ (due to the conditions assumed for the weighting translation function $f$ in Def.14):

Def. 16: Let WAF $=\langle\mathbf{A}, \mathbf{R}, \omega\rangle$. Let $f$ be a weighting translation function. An $\mathrm{AF}_{\mathrm{Vs}}$ induced by WAF using $f$ is a triplet $\langle\mathbf{A}, \mathbf{A T T}, \vec{\succeq}\rangle$ where ATT is the set of attack relations over $\mathbf{A}\langle\stackrel{1}{\rightarrow}, \ldots, \stackrel{n}{\rightarrow}\rangle$ defined by:

- $\underset{\rightarrow}{\rightarrow}=\{(A, B) \in \mathbf{R}$ such that $f(\omega(A), \omega(B))=i\}$ and

- $\succeq$ is the reflexive transitive closure of the binary relation on ATT defined by: $\stackrel{i}{\rightarrow} \underset{\succ}{\rightarrow}$ iff $i>j$.

\section{Qualitative vs quantitative setting}

So far we have two ways for building an $A F_{V s}$ from a given WAF:

- either applying Def.3 and then using Def.13 (which offers a more interesting ordering than Def.11),

- or using Def.16.

There is an important difference between these two constructions: in the first case, the resulting preference over attacks is a partial preordering, whereas, in the second case, it is a total one. Hopefully, these two ways are coherent, in the following sense: the second construction provides a valued $A F_{V S}$ in which the preference over attacks refines the preference obtained by the first construction.

Prop. 1: Let WAF $=\langle\mathbf{A}, \mathbf{R}, \omega\rangle$ be a weighted argumentation framework. Let PAF be the corresponding preference argumentation framework obtained from WAF using Def.3. Let $A_{\text {VS }}^{1}$ (resp. $A F_{\text {VS }}^{2}$ ) be the argumentation system with attacks of various strength obtained from PAF (resp. WAF) using Def.13 (resp. Def.16). $\vec{\succeq}_{1}$ (resp. $\vec{\succeq}_{2}$ ) denotes the associated preference relation between attacks. We have:

- $\forall(A, B),(C, D) \in \mathbf{R}$, if $(A, B) \vec{\succ}_{1}(C, D)$ then $(A, B) \vec{\succ}_{2}(C, D)$.

- $\forall(A, B),(C, D) \in \mathbf{R}$, if $(A, B) \vec{\sim}_{1}(C, D)$ then $(A, B) \vec{\sim}_{2}(C, D)$.

- $\forall(A, B),(C, D) \in \mathbf{R}$, if $(A, B) \vec{\succ}_{2}(C, D)$ then $(A, B) \vec{\succ}_{1}(C, D)$ or $(A, B) \vec{\varkappa}_{1}(C, D)$.

Proof

- Let $(A, B),(C, D) \in \mathbf{R}$ such that $(A, B) \vec{\succ}_{1}(C, D)$. Due to Def.12 and 13, it is sufficient to consider three situations:

1) $(A, B) \in \stackrel{i}{\rightarrow}$ and $(C, D) \in \stackrel{j}{\rightarrow}$ with $i<j$. For instance, we have $\omega(A)>\omega(B)(i=1)$ and $\omega(C)=\omega(D)$ $(j=2)$. In that case, due to the first and second items of Def.14, we have $f(\omega(A), \omega(B))>f(\omega(C), \omega(D))$ and then $(A, B) \vec{\succ}_{2}(C, D)$. The proof is similar for the other cases $(i=1$ and $j=4, i=2$ and $j=4)$.

2) $B=D,(A, B),(C, B) \in \stackrel{i}{\rightarrow}$ with $A \succ C$ (or equivalently $\omega(A)>\omega(C)$ ). For instance, we have $\omega(A)>\omega(B)$ and $\omega(C)>\omega(B)(i=1)$. In that case, due to the third item of Def.14, we have $f(\omega(A), \omega(B))>f(\omega(C), \omega(B))$ and then $(A, B) \vec{\succ}_{2}$ $(C, B)$. For the case $i=4$, we use the fourth item of Def.14.

3) $A=C,(A, B),(A, D) \in \stackrel{i}{\rightarrow}$ with $D \succ B$ (or equivalently $\omega(D)>\omega(B)$ ). For instance, we have $\omega(A)>\omega(B)$ and $\omega(A)>\omega(D)(i=1)$. In that case, due to the third item of Def.14, we have $f(\omega(A), \omega(B))>f(\omega(A), \omega(D))$ and then $(A, B) \vec{\succ}_{2}$ $(A, D)$. For the case $i=4$, we use the fourth item of Def.14.

- Let $(A, B),(C, D) \in \mathbf{R}$ such that $(A, B) \vec{\sim}_{1}(C, D)$. Due to Def.12 and 13, three different situations may occur.

1) $(A, B),(C, D) \in \stackrel{2}{\rightarrow}$. So $\omega(A)=\omega(B)$ and $\omega(C)=$ $\omega(D)$. In that case, due to the second item of Def 14, we have $f(\omega(A), \omega(B))=f(\omega(C), \omega(D))$ and then $(A, B) \vec{\sim}_{2}(C, D)$

2) $B=D,(A, B),(C, B) \in \stackrel{i}{\rightarrow}$ (i = 1 or 4) with $A \sim C$ (or equivalently $\omega(A)=\omega(C)$ ). Obviously, in that case, $f(\omega(A), \omega(B))=f(\omega(C), \omega(D))$ and then $(A, B) \vec{\sim}_{2}$ $(C, D)$.

3) $A=C,(A, B),(A, D) \in \stackrel{i}{\rightarrow}$ (i = 1 or 4$)$ with $D \sim B$ (or equivalently $\omega(D)=\omega(B)$ ). Obviously, in that case, $f(\omega(A), \omega(B))=f(\omega(C), \omega(D))$ and then $(A, B) \vec{\sim}_{2}$ $(C, D)$.

- Let $(A, B),(C, D) \in \mathbf{R}$ such that $(A, B) \vec{\succ}_{2}(C, D)$. Due to the first and second points of this proof, we cannot have $(C, D) \vec{\succ}_{1}(A, B)$, nor $(A, B) \vec{\sim}_{1}(C, D)$. So we must have either $(A, B) \vec{\succ}_{1}(C, D)$, or $(A, B) \vec{\not}_{1}(C, D)$.

\section{Examples}

In this section, our main definitions are illustrated on $A F=\langle\mathbf{A}, \mathbf{R}\rangle$ represented by the following graph: 


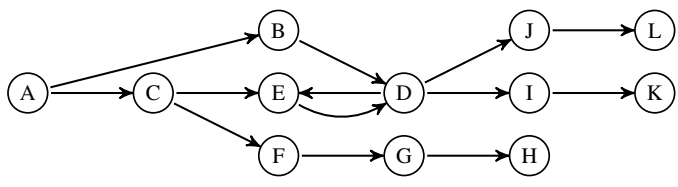

First, we consider the PAF $=\langle\mathbf{A}, \mathbf{R}, \succeq\rangle$ such that: $\begin{array}{lllll}A \succ B & D \succ I & J \succ L & B \sim D & F \not \approx G \\ C \succ E & D \succ J & J \succ I & C \sim F & D \not \approx E \\ C \succ A & I \succ K & & G \sim H & \end{array}$ The $A F_{\text {vs }}$ obtained with Def.11 is represented by:

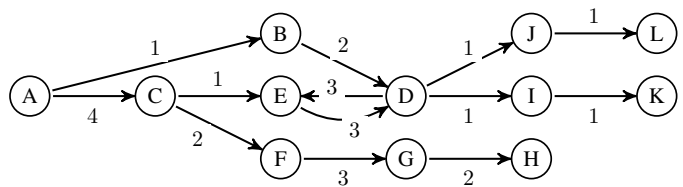

In this example, Arguments $E$ and $F$ are not vs-defended by $A$ against $C$, the attack from $A$ to $C$ being too weak; similarly, $H$ is not vs-defended by $F$ against $G$, the attack from $F$ to $G$ being too weak. Moreover, following Def.12, $(D, I) \vec{\succ}(D, J)$ (and this fact has no impact on the vsdefence). However, with Def.12, $(A, B),(C, E)$ and $(D, I)$ (for instance) are left incomparable.

Now, we consider the WAF $=\langle\mathbf{A}, \mathbf{R}, \omega\rangle$ with the following weighting function:

- $\omega(A)=0.7$

- $\omega(B)=\omega(D)=\omega(E)=0.6$

- $\omega(C)=\omega(F)=\omega(G)=\omega(H)=1$

- $\omega(J)=0.5$

- $\omega(I)=0.4$

- $\omega(K)=\omega(L)=0.3$

Using Def.15 with $\alpha=\beta=1^{10}$, the AF $\mathrm{Vs}_{\text {s }}$ obtained with Def.16 is represented by:

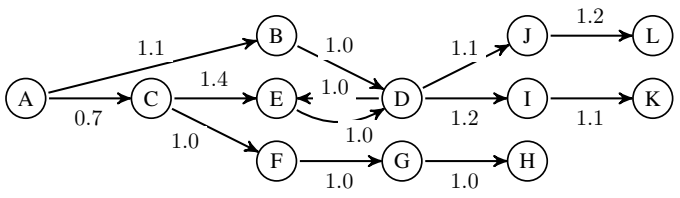

Here again, Arguments $E$ and $F$ are not $v s$-defended by $A$ against $C$, the attack from $A$ to $C$ being too weak. The case of $H$ is different: $H$ is vs-defended by $F$ against $G$ since Arguments $F$ and $G$ have been made equivalent. Note also that, due to Principles $\mathbf{P 4}{ }^{\prime}$ and $\mathbf{P 5}^{\prime}$, a difference between $K$ and $L$ appears: $L$ is no longer vs-defended. Moreover, this example illustrates Point 3 of Prop.1: with Def.16, we have $(C, E) \vec{\succ}(D, I) \vec{\succ}(A, B)$, whereas these attacks were incomparable with Def.12.

\section{A COMPARATIVE STUDY OF SEVERAL PROPOSALS}

In this section, we propose a comparative study of the different proposals presented so far for defining preferences

\footnotetext{
${ }^{10}$ So, the weighting translation function will be defined by $f_{11}:[0,1] \times$ $[0,1] \rightarrow[0,2]$ with $f_{11}(x, y)=(x-y)+1$.
}

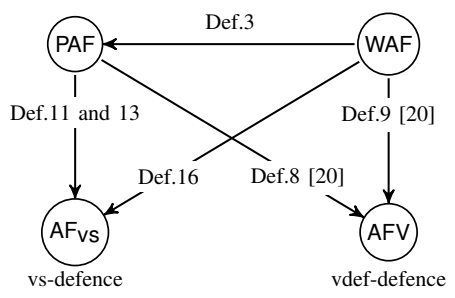

Figure 1. The different proposals for coping with preferences between arguments

over attacks from preferences over arguments. We are interested in two issues: the ordering between the attacks and the notion of defence. Figure 1 gives a synthetic representation of these different proposals.

\section{A. Proposals issued from a PAF}

Starting from a PAF, we compare the approach proposed by [20] given in Def.8 with our approach given in Def.11.

Table I shows how the set of attacks is partitioned.

\begin{tabular}{|c|c|c|c|}
\hline & \multirow{2}{*}{$\begin{array}{c}\text { Our approach } \\
\quad A \stackrel{i}{\rightarrow} B\end{array}$} & \multicolumn{2}{|c|}{ Approach of [20] } \\
\hline & & $\operatorname{Vdef}_{1}^{\succ}(A, B)$ & $\operatorname{Vdef}_{2}^{\succ}(A, B)$ \\
\hline$A \succ B$ & $A \stackrel{1}{\rightarrow} B$ & 1 & 1 \\
\hline$A \sim B$ & $A \stackrel{2}{\rightarrow} B$ & 1 & $1 / 2$ \\
\hline$A \not \approx B$ & $A \stackrel{3}{\rightarrow} B$ & 1 & $1 / 2$ \\
\hline$B \succ A$ & $A \stackrel{4}{\rightarrow} B$ & 0 & 0 \\
\hline & 4 classes & 2 classes & 3 classes \\
\hline
\end{tabular}

Classes of attacks in the Case of PAF

The main difference lies in the treatment of attacks $(A, B)$ such that $B \succ A$. With the weighted attacks of [20], all these attacks are removed (since the weight equals to 0 ), whereas we keep these attacks. Moreover, our approach enables to distinguish the attacks between equivalent arguments with those between incomparable arguments.

Then we are interested in the defence provided by $C$ for $A$ against $B$. Table II shows the different cases. Three situations are worth-noticing (in bold in the table). They highlight the fact that our approach provides a finer partition of the set of attacks.

Note that considering Def.13 instead of Def.11 would not modify the comparison, since applying Def.13 does not modify the hierarchy of the classes of attacks. It only adds preferences between attacks of the same class. Moreover, the ordering defined by Def.13 has no impact on the vs-defence.

\section{B. Proposals issued from a WAF}

Starting from a WAF, we compare the approach proposed by [20] given in Def.9 with our proposal for building an $\mathrm{AF}_{\mathrm{Vs}}$. As said in Section III-C, we can apply either Def.3 and then Def.13, or Def.16. We successively consider both 


\begin{tabular}{|c|c|c|c|}
\hline & \multirow{2}{*}{$\begin{array}{c}\text { Our approach } \\
\text { vs-defence }\end{array}$} & \multicolumn{2}{|c|}{ Approach of [20] } \\
\hline & & $\operatorname{Vdef}_{1}^{\succ}$-defence & Vde $f_{2}^{\succ}$-defence \\
\hline$C \succ B \succ A$ & yes & yes & $\overline{\text { yes }}$ \\
\hline$C \succ B \sim A$ & yes & yes & yes \\
\hline$C \succ B \not \approx A$ & yes & yes & yes \\
\hline$C \succ B \prec A$ & yes & \multicolumn{2}{|c|}{ yes, since no attack on $A$} \\
\hline$C \sim B \succ A$ & no & $\overline{\mathbf{y e s}}$ & $\overline{\text { no }}$ \\
\hline$C \sim B \sim A$ & yes & yes & yes \\
\hline$C \sim B \not \approx A$ & yes & yes & yes \\
\hline$C \sim B \prec A$ & yes & \multicolumn{2}{|c|}{ yes, since no attack on $A$} \\
\hline$C \not \approx B \succ A$ & no & yes & no \\
\hline$C \not \approx B \sim A$ & no & yes & yes \\
\hline$C \not \approx B \not \approx A$ & yes & yes & yes \\
\hline$C \not \approx B \prec A$ & yes & \multicolumn{2}{|c|}{ yes, since no attack on $A$} \\
\hline$C \prec B \succ A$ & no & \multicolumn{2}{|c|}{ no, since no attack on $B$} \\
\hline$C \prec B \sim A$ & no & \multicolumn{2}{|c|}{ no, since no attack on $B$} \\
\hline$C \prec B \not \approx A$ & no & \multicolumn{2}{|c|}{ no, since no attack on $B$} \\
\hline$C \prec B \prec A$ & yes & \multicolumn{2}{|c|}{$\begin{array}{l}\text { yes, since no attack on } B \\
\text { and no attack on } A\end{array}$} \\
\hline
\end{tabular}

Table II

Defence in the CASE of PAF: Let $(C, B),(B, A) \in \mathbf{R}$, IS $C$ A DEFENDER OF $A$ AGAINST $B$ ?

cases. Table III partially shows how the attacks are ordered in the quantitative setting (we only consider $\operatorname{Vde} f_{1}^{\omega}$ and Vde $f_{2}^{\omega}$, since the other definitions correspond to $\operatorname{Vde} f_{1}^{\succ}$ and $\operatorname{Vde} f_{2}^{\succ}$ ).

\begin{tabular}{|c|c|c|c|}
\hline & \multirow{2}{*}{$\begin{array}{l}\text { Our prop. } \\
A \stackrel{i}{\rightarrow} B\end{array}$} & \multicolumn{2}{|c|}{ Proposition of [20] } \\
\hline & & $\operatorname{Vdef}_{1}^{\omega}(A, B)$ & $\operatorname{Vdef}_{2}^{\omega}(A, B)$ \\
\hline $\begin{array}{c}\omega(A)>\omega(B) \\
\text { so } A \succ B\end{array}$ & $A \stackrel{1}{\rightarrow} B$ & $\begin{array}{c}\omega(A)-\omega(B) \\
\quad \in] 0 ; 1]\end{array}$ & 1 \\
\hline $\begin{array}{c}\omega(A)=\omega(B) \\
\quad \text { so } A \sim B \\
\end{array}$ & $A \stackrel{2}{\rightarrow} B$ & 0 & 1 \\
\hline $\begin{array}{c}\omega(A)<\omega(B) \\
\text { so } B \succ A\end{array}$ & $A \stackrel{4}{\rightarrow} B$ & 0 & $\begin{array}{c}1-(\omega(B)-\omega(A)) \\
\in[0 ; 1[\end{array}$ \\
\hline & $\begin{array}{c}3 \text { classes } \\
\text { (classes 1, } \\
3 \text { refined) }\end{array}$ & $\begin{array}{l}2 \text { classes } \\
\text { with } 1 \\
\text { refined class }\end{array}$ & $\begin{array}{l}2 \text { classes } \\
\text { with } 1 \\
\text { refined class }\end{array}$ \\
\hline
\end{tabular}

Table III

PREFERENCES ON ATTACKS IN THE CASE OF WAF

Note that with our approach, the hierarchy between the classes of attacks remains unchanged (the class $\stackrel{3}{\rightarrow}$ has been removed since there are no incomparable arguments in a WAF). Moreover, due to Def.13, the classes $\stackrel{1}{\rightarrow}$ and $\stackrel{4}{\rightarrow}$ are partially ordered. Let us prove that in the class $\stackrel{1}{\rightarrow}$ (resp. the class $\stackrel{4}{\rightarrow}$ ) this partial ordering is extended by the ordering associated with Vde $f_{1}^{\omega}$ (resp. Vde $f_{2}^{\omega}$ ).

Let $(A, B),(C, D) \in \stackrel{1}{\rightarrow}$ (resp. $\stackrel{4}{\rightarrow}$ ) such that $(A, B) \vec{\succ}$ $(C, D)$. Then, it must be the case that $B=D$ or $A=C$. Moreover, due to Def.13, if $(A, B) \vec{\succ}(C, B)$, we have necessarily $A \succ C$, and if $(A, B) \vec{\succ}(A, D)$, we have necessarily $D \succ B$. So,

- With $A \stackrel{1}{\rightarrow} B, C \stackrel{1}{\rightarrow} B,(A, B) \vec{\succ}(C, B)$ implies $\omega(A)>\omega(C)$ and then $\omega(A)-\omega(B)>\omega(C)-\omega(B)$.
So $\operatorname{Vdef}_{1}^{\omega}(A, B)>\operatorname{Vdef}_{1}^{\omega}(C, B)$.

- With $A \stackrel{1}{\rightarrow} B, A \stackrel{1}{\rightarrow} D,(A, B) \vec{\succ}(A, D)$ implies $\omega(D)>\omega(B)$ and then $\omega(A)-\omega(B)>\omega(A)-\omega(D)$.

- With $A \stackrel{4}{\rightarrow} B, C \stackrel{4}{\rightarrow} B$, $(A, B) \stackrel{\vec{\succ}}{ }(C, B)$ implies $\omega(A)>\omega(C)$ and then $1-(\omega(B)-\omega(A))>1-$ $\left(\omega(B)-\omega(C)\right.$. So $\operatorname{Vdef}_{2}^{\omega}(A, B)>\operatorname{Vdef}_{2}^{\omega}(C, B)$.

- With $A \stackrel{4}{\rightarrow} B, A \stackrel{4}{\rightarrow} D,(A, B) \vec{\succ}(A, D)$ implies $\omega(D)>\omega(B)$ and then $1-(\omega(B)-\omega(A))>1-$ $(\omega(D)-\omega(A))$.

Note also that, as in the case of PAF, some attacks are removed in [20] (particularly with $\operatorname{Vdef}_{1}^{\omega}$ ).

Then, Table IV synthetizes the results about defence in the different approaches. Some particular cases (in bold in the table) are identified and described below.

\begin{tabular}{|c|c|c|c|}
\hline & \multirow{2}{*}{$\begin{array}{l}\text { Our approach } \\
\text { vs-defence }\end{array}$} & \multicolumn{2}{|c|}{ Approach of [20] } \\
\hline & & Vdef ${ }_{1}^{\omega}$-defence & Vdef ${ }_{2}^{\omega}$-defence \\
\hline $\begin{array}{l}\omega(C)>\omega(B) \\
\omega(B)>\omega(A)\end{array}$ & yes & Case 1 & yes \\
\hline $\begin{array}{l}\omega(C)>\omega(B) \\
\omega(B)=\omega(A)\end{array}$ & yes & yes (Note 1) & yes \\
\hline $\begin{array}{l}\omega(C)>\omega(B) \\
\omega(B)<\omega(A)\end{array}$ & yes & yes (Note 1) & yes \\
\hline $\begin{array}{l}\omega(C)=\omega(B) \\
\omega(B)>\omega(A)\end{array}$ & no & no (Note 1$)$ & yes (Case 3) \\
\hline $\begin{array}{l}\omega(C)=\omega(B) \\
\omega(B)=\omega(A)\end{array}$ & yes & yes (Note 1) & yes \\
\hline $\begin{array}{l}\omega(C)=\omega(B) \\
\omega(B)<\omega(A)\end{array}$ & yes & yes (Note 1) & yes \\
\hline $\begin{array}{l}\omega(C)<\omega(B) \\
\omega(B)>\omega(A)\end{array}$ & no & no (Note 1) & no \\
\hline $\begin{array}{l}\omega(C)<\omega(B) \\
\omega(B)=\omega(A)\end{array}$ & no & yes (Note 1) & no \\
\hline $\begin{array}{l}\omega(C)<\omega(B) \\
\omega(B)<\omega(A)\end{array}$ & yes & yes (Note 1) & Case 2 \\
\hline
\end{tabular}

Table IV

Defence in the CASE of WAF: Let $(C, B),(B, A) \in \mathbf{R}$, IS $C$ A DEFENDER OF $A$ AGAINST $B$ ?

Note 1: With Vdef ${ }_{1}^{\omega}$, attacks $(D, E)$ such that $\omega(D) \ngtr$ $\omega(E)$ are not considered. So in many cases, either $A$ is defended since the attack from $B$ to $A$ does not exist or $A$ cannot be defended since the attack from $C$ to $B$ does not exist.

Case 1: In this case, $C$ Vdef $f_{1}^{\omega}$-defends $A$ against $B$ except if $(\omega(B)-\omega(A))>(\omega(C)-\omega(B))$. In contrast, $C$ always vs-defends $A$ against $B$.

Case 2: In this case, $C \operatorname{Vdef}_{2}^{\omega}$-defends $A$ against $B$ except if $(\omega(B)-\omega(C))>(\omega(A)-\omega(B))$. In contrast, $C$ always vs-defends $A$ against $B$.

Case 3: As Vde $f_{2}^{\omega}$ does not discriminate between an attack $(A, B)$ with $A \succ B$ and an attack $(A, B)$ with $A \sim B$, if $(\omega(B)=\omega(C))>\omega(A), C \operatorname{Vdef}_{2}^{\omega}$-defends $A$ against $B$. In contrast, we have $B \stackrel{1}{\rightarrow} A$ and $C \stackrel{2}{\rightarrow} B$, so $C$ does not vs-defend $A$ against $B$.

Finally, let us consider the valued $A F_{v s}$ obtained with 
Def.16. Due to the conditions stated in Def.14, and the definition of $\operatorname{Vdef} f_{1}^{\omega}$ and $\operatorname{Vde} f_{2}^{\omega}$, it is easy to see that:

- With $\omega(A)>\omega(B)$ and $\omega(C)>\omega(D)$, if $\operatorname{Vdef}_{1}^{\omega}(A, B)>\operatorname{Vdef}_{1}^{\omega}(C, D)$, then $f(\omega(A), \omega(B))>f(\omega(C), \omega(D))$

- With $\omega(A)<\omega(B)$ and $\omega(C)<\omega(D)$, if $\operatorname{Vdef}_{2}^{\omega}(A, B)>\operatorname{Vdef}_{2}^{\omega}(C, D)$, then $f(\omega(A), \omega(B))>f(\omega(C), \omega(D))$

In other words, the valued $A_{\mathrm{Vs}}$ obtained with Def.16 allows for a total preordering on $\mathbf{R}$ that extends the $\mathrm{Vde} \mathrm{f}_{1}^{\omega}$ based ordering on the attacks $(A, B)$ such that $A \succ B$ and the Vdef $f_{2}^{\omega}$-based ordering on the attacks $(A, B)$ such that $B \succ A$ :

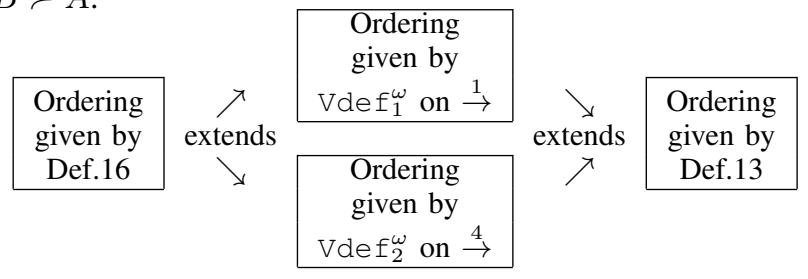

V. CONCLUSION

In this paper, we have investigated the relationships between preferences over arguments and preferences over attacks in abstract argumentation. We have laid down some principles which should govern the definition of preferences over attacks from preferences over arguments. Following these principles, we have proposed different instantiations of an AF vs (argumentation framework with attacks of various strength), when preferences over arguments are available. We have considered qualitative as well as quantitative approaches for expressing preferences. Then, we have compared our proposal with related works, particularly regarding the conditions in which the defense holds. This work is a first contribution towards a better handling of preferences in argumentation. The proposed principles will have to be validated by considering how preferences over arguments can be obtained, when arguments have an internal structure (for instance, when they are built from pieces of knowledge). Another issue for future work concerns the computation of acceptable sets of arguments, when arguing with preferences.

\section{REFERENCES}

[1] P. Krause, S. Ambler, M. Elvang, and J. Fox, "A logic of argumentation for reasoning under uncertainty," Computational Intelligence, vol. 11 (1), pp. 113-131, 1995.

[2] H. Prakken and G. Vreeswijk, "Logics for defeasible argumentation," in Handbook of Philosophical Logic. Kluwer Academic, 2002, vol. 4, pp. 218-319.

[3] L. Amgoud and C. Cayrol, "Inferring from inconsistency in preference-based argumentation frameworks," Journal of Automated Reasoning, vol. 29, pp. 125-169, 2002.
[4] A. C. Kakas and P. Moraïtis, "Argumentation based decision making for autonomous agents," in Proc. of AAMAS, 2003, pp. 883-890.

[5] L. Amgoud, Y. Dimopoulos, and P. Moraïtis, "A unified and general framework dor argumentation-based negotiation," in Proc. of AAMAS, 2007, pp. 963-970.

[6] P. M. Dung, "On the acceptability of arguments and its fundamental role in nonmonotonic reasoning, logic programming and n-person games," Artificial Intelligence, vol. 77, pp. 321357, 1995.

[7] L. Amgoud and C. Cayrol, "A reasoning model based on the production of acceptable arguments," Annals of Mathematics and Artificial Intelligence, vol. 34, pp. 197-216, 2002.

[8] T. Bench-Capon, "Persuasion in practical argument using value-based argumentation frameworks," Journal of Logic and Computation, vol. 13, no. 3, pp. 429-448, 2003.

[9] A. Z. Wyner and T. J. Bench-Capon, "Towards an extensible argumentation system," in Proc of ECSQARU (LNAI 4724), 2007, pp. 283-294.

[10] S. Modgil, "Reasoning about preferences in argumentation frameworks," Artificial Intelligence, vol. 173, pp. 901-934, 2009.

[11] M. Elvang-Gøransson and A. Hunter, "Argumentative logics: Reasoning with classically inconsistent information," Data and Knowledge Engineering, vol. 16, pp. 125-145, 1995.

[12] P. Besnard and A. Hunter, Elements of argumentation. MIT Press, 2008.

[13] D. C. Martinez, A. J. Garcia, and G. R. Simari, "Strong and weak forms of abstract argument defense," in Proc of COMMA, 2008, pp. 216-227.

[14] F. A. Tohmé, G. A. Bodanza, and G. R. Simari, "Aggregation of attack relations: a social-choice theoretical analysis of defeasibility criteria," in Proc of FoIKS (LNCS 4932), 2008, pp. 8-23.

[15] H. Barringer, D. Gabbay, and J. Woods, "Temporal dynamics of support and attack networks : From argumentation to zoology," in Mechanizing Mathematical Reasoning. LNAI 2605. Springer Verlag, 2005, pp. 59-98.

[16] P. E. Dunne, A. Hunter, P. McBurney, S. Parsons, and M. Wooldridge, "Inconsistency tolerance in weighted argument systems," in Proc of AAMAS, 2009, pp. 851-858.

[17] D. C. Martinez, A. J. Garcia, and G. R. Simari, "An abstract argumentation framework with varied-strength attacks," in Proc of KR, 2008, pp. 135-143.

[18] C. Cayrol, C. Devred, and M.-C. Lagasquie-Schiex, "Acceptability semantics accounting for strength of attacks in argumentation," in Proc. of ECAI, 2010, pp. 995-996.

[19] — "Dialectical proofs accounting for strength of attacks," in Proc of ICTAI, 2010, pp. 207-214.

[20] S. Kaci and C. Labreuche, "Arguing with valued preference relations," in Proc. of ECSQARU, 2011, pp. 62-73. 